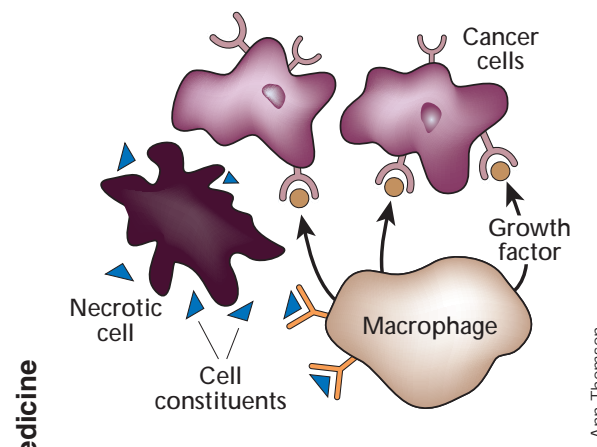

Figure 2 Beyond the grave. Once a cancer reaches a certain size, its core no longer receives sufficient amounts of nutrients. This results in necrosis of some cancer cells. The release of normal cellular constituents by the necrotic cell can lead to activation of stromal- or tumor-infiltrating macrophages which produce growth factors, such as IL-6, that stimulate the proliferation of surviving cancer cells.

by a product of activated inflammatory cells that migrated into the tumor via IL-8-induced chemotaxis (Fig. 1). Indeed, treatment of tumorbearing mice with an antibody that depletes myeloid cells also results in reduced tumor growth and angiogenesis ${ }^{5}$. Importantly, the antitumorigenic effect of the inhibitory antibodies used by Sparmann and Bar-Sagi was executed at the level of tumor angiogenesis rather than directly at tumor-cell proliferation or survival ${ }^{5}$.
Is IL-8 the only proinflammatory molecule that links oncogene activation, inflammation and tumor growth? The answer is probably not. Another candidate, whose synthesis is regulated in a similar manner to IL-8, is the proinflammatory cytokine IL-6 (ref.7). Recent work has shown that IL-6 produced by tumor-infiltrating T cells, dendritic cells and epithelial cells drives the growth of colitis-associated cancer (CAC) induced by administration of the procarcinogen azoxymethane and the irritant dextrane sulphate salt (DSS $)^{10}$. Administration of DSS results in an inflammatory response that leads to activation of transcription factor NF- $\kappa \mathrm{B}$, an activator of IL- 6 gene transcription $^{11}$. Deletion of the gene that encodes I $\mathrm{B} B$ kinase $\beta(\operatorname{IKK} \beta)$, a protein kinase that activates NF- $\kappa B$, in myeloid cells also reduces the growth of $\mathrm{CAC}^{11}$.

Whereas the major source of IL-6 expression in already-developed CAC appears to be tumorinfiltrating $\mathrm{T}$ cells ${ }^{10}$, during the tumor promotion stage IL-6 is mainly produced by myeloid cells ${ }^{11}$. Although the source of IL- 6 and other growth factors may change during tumor progression, it is clear that an interaction between transformed epithelial cells and nontransformed inflammatory cells may lead to direct recruitment and eventual activation of endothelial cells. A likely scenario entails recruitment of endothelial cells by a product of activated myeloid and lymphoid cells that strongly influences tumor development and progression.

The molecular nature of the interaction between tumor cells and hematopoietic-derived cells may vary from one case of cancer to another.
Sparmann and Bar-Sagi outline how chemokines like IL-8 produced by cancer cells in response to oncogene activation recruit and activate myeloid cells. But in other cases, such as CAC, the myeloid and lymphoid cells may already reside in close proximity to the developing cancer. In CAC, these cells are probably activated through an inflammatory chain reaction initiated by invasion of enteric bacteria into the lamina propria ${ }^{11}$. In other cases, it is possible that the growing tumor being starved of blood supply may undergo necrosis and the necrotic cell products lead to activation of adjacent macrophages or other myeloid cells — which then secrete growth factors that promote further tumor growth and angiogenesis (Fig. 2).

Regardless of detail, it is likely that better understanding of such interactions will pave the way for new therapies that will either prevent activation of inflammatory cells by cancerderived products, or block the ability of growth factors produced by inflammatory cells to stimulate tumor angiogenesis, growth and survival.

1. Coussens, L.M. \& Werb, Z. Nature 420, 860-867 (2002).

2. Balkwill, F. \& Mantovani, A. Lancet 357, 539-545 (2001).

3. Reedy, J. Clio Medica 10, 227-238 (1975).

4. Dvorak, H.F. N. Engl. J. Med. 315, 1650-1659 (1986).

5. Sparmann, A. \& Bar-Sagi, D. Cancer Cell 6, 447-458 (2004).

6. Karin, M. J. Biol. Chem. 270, 16483-16486 (1995).

7. Matsusaka, T. et al. Proc. Natl. Acad. Sci. USA 90, 1019310197 (1993)

8. Yasumoto, K. et al., J. Biol. Chem. 267, 22506-22511 (1992).

9. Matsushima, K. et al., J. Exp. Med. 167, 1883-1893 (1988).

10. Becker, C. et al. Immunity 21, 491-501 (2004)

11. Greten, F.R. et al. Cell 118 285-296 (2004).

\title{
Inflammation and migration
}

Cells derived from the bone marrow spawn the gastric tumors induced by Helicobacter pylori, suggest findings by JeanMarie Houghton et al. in the 26 November issue of Science $(\mathbf{3 0 6}, 1568-1571 ; 2004)$. The results contradict the thinking that such tumors arise from the stomach tissue, and mesh with findings that bone marrow-derived cells are recruited to sites of inflammation.

To investigate the source of gastric tumors, the researchers used a mouse strain that develops inflammation and then cancer when infected with $H$. felis. The researchers irradiated the mice to eliminate their bone marrow, and then transplanted the mice with marrow cells expressing $\beta$-galactosidase. In the absence of Helicobacter infection, only a few bone marrow-derived cells homed to the stomach tissues - and these were white blood cells (blue; inset). In response to Helicobacter infection, which induces inflammation, the bone marrow cells engrafted into the stomach lining (main image). The engrafted cells began to differentiate, resembling gastric epithelial cells. But the cells were not quite normal-they grew quickly, had distorted shapes, and eventually gave rise to cancerous tumors.

One question still not completely resolved is whether the tumors arose through fusion of cells in the stomach with bone marrow cells-which have a tendency to fuse to other cells. In this study, experiments counting chromosome numbers in cells expressing $\beta$-galactosidase suggested that fusion did not occur.

-Charlotte Schubert

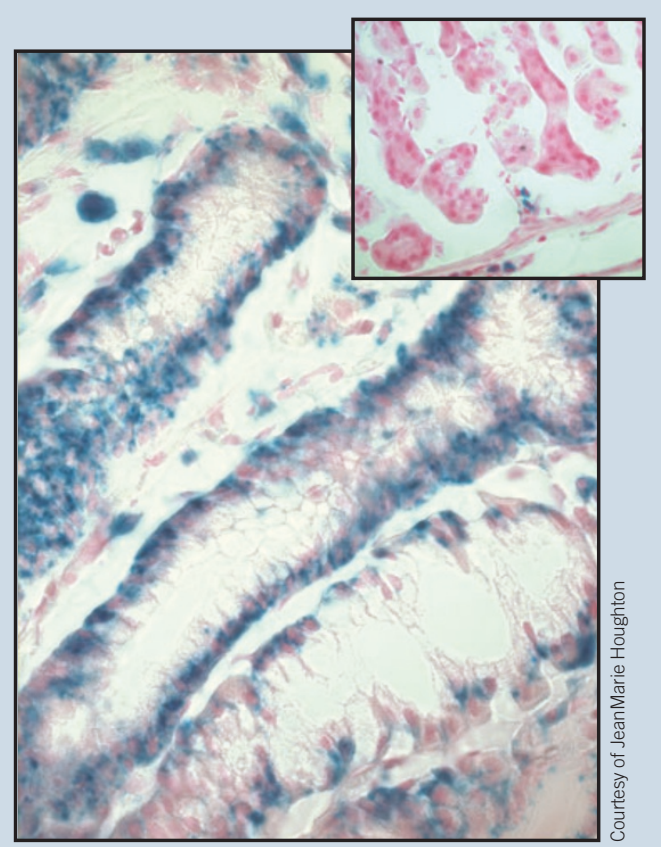

